\title{
On the Number of Membranes in Unary P Systems
}

\author{
Rudolf Freund * \\ Fakultät für Informatik - Technische Universität Wien \\ Favoritenstraße 9-11 - A-1040 Wien - Austria \\ rudi@emcc. at \\ Andreas Klein \\ Department of Pure Mathematics and Computer Algebra - Ghent University \\ Krijgslaan 281-S22 - B-9000 Ghent - Belgium \\ klein@cage.ugent.be \\ Martin Kutrib \\ Institut für Informatik - Universität Giessen \\ Arndtstraße 2 - D-35392 Giessen - Germany \\ kutrib@informatik. uni-giessen.de
}

\begin{abstract}
We consider P systems with a linear membrane structure working on objects over a unary alphabet using sets of rules resembling homomorphisms. Such a restricted variant of P systems allows for a unique minimal representation of the generated unary language and in that way for an effective solution of the equivalence problem. Moreover, we examine the descriptional complexity of unary $\mathrm{P}$ systems with respect to the number of membranes.
\end{abstract}

\section{Introduction}

One decade ago, inspired by biological systems to be found in nature, Gheorghe Păun introduced membrane systems (e.g., see [4]) as a class of distributed parallel computing models, originally working on multisets of objects. The main feature of membrane systems (soon called P systems in the literature) are the hierarchically structured membranes embedded in the outermost skin membrane. Every membrane encloses a region possibly containing other membranes as well as multisets of specific objects that evolve according to evolution rules associated with the regions. A computation is a sequence of consecutive configurations of the system evolving by applying the evolution rules in parallel to the objects in every region in a maximal manner. The vector of the multiplicities of objects present in the specified output membrane in the final configuration of a halting computation is considered to be the result of such a computation; in that way, a membrane system computes a set of vectors of natural numbers.

Many variants of $\mathrm{P}$ systems are able to characterize the family of recursively enumerable sets of vectors of natural numbers (the Parikh sets associated with recursively enumerable languages). The first detailed definitions and results can be found in [4], and an overview of many variants is given in [6]. The first monograph on membrane systems [5] shows the great variety and potentials of $\mathrm{P}$ systems; for the actual state of the art in membrane systems we refer the interested reader to the P page [9].

In [7] it is shown that special variants of $\mathrm{P}$ systems with the underlying membrane structure being only a linear tree can be computationally complete when using adequate evolution rules. In [3], several quite simple variants of such $\mathrm{P}$ systems were investigated that allowed for establishing an infinite hierarchy with respect to the number of membranes, which usually cannot be the case for computationally

* corresponding author

J. Dassow, G. Pighizzini, B. Truthe (Eds.): 11th International Workshop on Descriptional Complexity of Formal Systems (DCFS 2009)

EPTCS 3, 2009, pp. 101-109 doi 10.4204/EPTCS.3.9

(c) R. Freund, A. Klein, M. Kutrib 
complete variants of $\mathrm{P}$ systems. These special variants of $\mathrm{P}$ systems were inspired by the tree systems of morphisms (introduced in [2]) and the compound Lindenmayer systems (investigated in [1]). In this paper, we consider those variants as introduced in [3], yet especially over a unary alphabet, and investigate the descriptional complexity of the unary languages generated by such systems.

The rest of the paper is organized as follows: In the second section we give some preliminary definitions of notions needed in this paper and then define the special variant of self-reproducing $\mathrm{P}$ systems to be investigated in the succeeding sections; moreover, we also construct a suitable example and prove some first results. In the third section, we show how to construct a unique minimal representation of the unary language generated by a unary $\mathrm{P}$ system, which also allows us to show the decidability of the equivalence problem for unary $\mathrm{P}$ systems in the succeeding section. The descriptional complexity of unary $\mathrm{P}$ systems with respect to the number of membranes is investigated in the fifth section.

\section{A specific variant of $P$ systems}

In this section, after having specified some notions, we introduce the main variant of $\mathrm{P}$ systems investigated in this paper, i. e., $\mathrm{P}$ systems with a linear membrane structure and rules mapping each object to powers of itself. Moreover, we give an illustrative example and prove two useful lemmas valid for this specific variant of $\mathrm{P}$ systems.

\subsection{Preliminaries}

For the basic notions of formal language theory we refer the reader to monographs such as [8]. We just mention some specific notions used throughout the paper: The empty string is denoted by $\lambda$, and for the length of a string $w$ we write $|w|$. The set of mappings from a set $M$ to a set $N$ is denoted by $N^{M}$. We denote the set of positive integers by $\mathbb{N}_{+}$and the set of non-negative integers by $\mathbb{N} ; \mathbb{N}^{n}$ denotes the set of vectors $\left(x_{1}, \ldots, x_{n}\right)$ with $x_{i} \in \mathbb{N}, 1 \leq i \leq n$, and for $\left(x_{1}, \ldots, x_{n}\right) \in \mathbb{N}^{n},\left(x_{1}, \ldots, x_{n}\right)^{T}$ denotes the corresponding transposed vector. Moreover, we write $\mathbb{P}$ for the set of prime numbers, and throughout the paper, $p_{i}$ denotes the $i$-th prime number, i. e., $p_{1}=2, p_{2}=3, p_{3}=5, \ldots$

\subsection{Self-reproducing P systems}

Now let us consider the following very special variant of P systems, where

- the underlying membrane structure is a linear tree $\left[0\left[1 \ldots[n]_{n} \ldots\right]_{1}\right]_{0}$;

- the only axiom is in the innermost membrane;

- except for the skin membrane (labeled by 0 ), each region $i \geq 1$ contains the membrane dissolving operation $\delta$ as well as for each object $a$ exactly one (noncooperative) rule of the special form $a \rightarrow a^{m(i, a)}$ for some $m(i, a)>0$;

- there are no rules in the skin membrane;

- the result of a computation is the multiset finally appearing in the skin membrane (which constitutes a halting computation).

There are no additional features used when applying the (noncooperative) rules of the special form $a \rightarrow a^{m(i, a)}$ in a maximally parallel manner; for example, there are no priority relations among the rules. 
The movement of objects from the innermost membrane to the skin membrane only happens by dissolving one membrane after the other one using the membrane dissolving operation $\delta$ which may be chosen at any time for a membrane containing at least one object.

These special $\mathrm{P}$ systems as described above were introduced in [3] and had been inspired by the tree systems of morphisms (introduced in [2]) and the compound Lindenmayer systems (investigated in [1]). In the case of linear trees, these tree systems with morphisms intuitively correspond with the self-reproducing deterministic linear P systems as defined above. The main difference is the (additional) skin membrane, which only serves for terminating the computation and collecting its result.

Due to this correspondence with tree systems with morphisms, we can easily describe a P system of the special form as defined above in the following way:

$$
\Pi=\left(V, h_{1}, \ldots, h_{n}, w\right)
$$

where $V$ is an alphabet, $n$ is the height of the linear tree describing the membrane structure, $w$ is the axiom over $V$, and the $h_{i}$ are the homomorphisms defined by the rules of the form $a \rightarrow a^{m(i, a)}$ in region $i$. As each object is mapped to powers of itself only, we call these $\mathrm{P}$ systems self-reproducing.

As it is easy to see, the language generated by a self-reproducing P system

$$
\Pi=\left(V, h_{1}, \ldots, h_{n}, w\right)
$$

can be written as

$$
h_{1}^{*}\left(h_{2}^{*} \cdots\left(h_{n}^{*}(w)\right) \cdots\right)
$$

in other words,

$$
L_{*}(\Pi)=\left\{h_{1}^{m_{1}}\left(h_{2}^{m_{2}} \cdots\left(h_{n}^{m_{n}}(w)\right) \cdots\right) \mid m_{i} \geq 0,1 \leq i \leq n\right\} .
$$

We may also demand that every homomorphism has to be applied at least once before the corresponding membrane may be dissolved; in that case, the corresponding language generated by $\Pi=\left(V, h_{1}, \ldots, h_{n}, w\right)$ can be written as

$$
h_{1}^{+}\left(h_{2}^{+} \cdots\left(h_{n}^{+}(w)\right) \cdots\right),
$$

in other words,

$$
L_{+}(\Pi)=\left\{h_{1}^{m_{1}}\left(h_{2}^{m_{2}} \cdots\left(h_{n}^{m_{n}}(w)\right) \cdots\right) \mid m_{i} \geq 1,1 \leq i \leq n\right\} .
$$

\subsection{An illustrative example and first results}

Example 1. For each $n \geq 1$, consider the language

$$
L(n)=\left\{a_{1}^{p_{1}^{m_{1}}} \ldots a_{n}^{p_{n}^{m_{n}}} \mid m_{i} \geq 0,1 \leq i \leq n\right\}
$$

where $p_{i}$ denotes the $i$-th prime number.

$L(n)$ is generated by the $\mathrm{P}$ system

$$
\Pi(n)=\left(V, h_{1}, \ldots, h_{n}, w\right)
$$

with $V=\left\{a_{1}, \ldots, a_{n}\right\}, w=a_{1} \ldots a_{n}, h_{i}\left(a_{i}\right)=a_{i}^{p_{i}}$ and $h_{i}\left(a_{k}\right)=a_{k}$ for $k \neq i, 1 \leq k \leq n, 1 \leq i \leq n$.

Obviously, $a_{1}^{p_{1}^{m_{1}}} \ldots a_{n}^{p_{n}^{m_{n}}}$ is obtained as $h_{1}^{m_{1}}\left(\cdots\left(h_{n}^{m_{n}}\left(a_{1} \ldots a_{n}\right)\right) \cdots\right)$; moreover, only elements from $L(n)$ can be generated by $\Pi(n)$, hence, $L(n)=L_{*}(\Pi(n))$. Finally, we observe that

$$
L_{+}(\Pi(n))=\left\{a_{1}^{p_{1}^{m_{1}}} \ldots a_{n}^{p_{n}^{m_{n}}} \mid m_{i} \geq 1,1 \leq i \leq n\right\} .
$$


As it was shown in [3], $L(n)$ cannot be generated by a self-reproducing $\mathrm{P}$ system with less than $n$ homomorphisms (or equivalently, less than $n+1$ membranes), i. e., these variants of $\mathrm{P}$ systems establish an infinite hierarchy with respect to the number of membranes.

We now establish two simple but nevertheless useful lemmas. The first observation is that the ordering of the homomorphisms is irrelevant (which directly follows from the commutativity of multiplication):

Lemma 2. Let $\Pi=\left(V, h_{1}, \ldots, h_{n}, w\right)$ be a self-reproducing $P$ system, $u$ be a permutation of $1, \ldots, n$, and $\Pi^{\prime}=\left(V, h_{u(1)}, \ldots, h_{u(n)}, w\right)$. Then $L_{*}(\Pi)=L_{*}\left(\Pi^{\prime}\right)$ as well as $L_{+}(\Pi)=L_{+}\left(\Pi^{\prime}\right)$.

Proof: Let $a \in V$ and let $|w|_{a}$ denote the number of objects $a$ in the axiom $w$ of $\Pi$; then obviously

$$
h_{u(1)}^{m_{u(1)}}\left(h_{u(2)}^{m_{u(2)}} \cdots\left(h_{u(n)}^{m_{u(n)}}\left(a^{|w|_{a}}\right)\right) \cdots\right)=h_{1}^{m_{1}}\left(h_{2}^{m_{2}} \cdots\left(h_{n}^{m_{n}}\left(a^{|w|_{a}}\right)\right) \cdots\right),
$$

as, due to the commutativity of multiplication, $\left(a^{m}\right)^{k}=a^{m k}=a^{k m}=\left(a^{k}\right)^{m}$ for all $k, m \geq 1$.

Using Lemma 2, next we show that we may move the first application of every homomorphism into the axiom, i. e., for every self-reproducing $\mathrm{P}$ system $\Pi$ there is an equivalent self-reproducing $\mathrm{P}$ system $\Pi^{\prime}$ such that $L_{+}(\Pi)=L_{*}\left(\Pi^{\prime}\right)$.

Lemma 3. Let $\Pi$ be a self-reproducing $P$ system. Then we can effectively construct a self-reproducing $P$ system $\Pi^{\prime}$ such that $L_{+}(\Pi)=L_{*}\left(\Pi^{\prime}\right)$.

Proof: For $\Pi=\left(V, h_{1}, \ldots, h_{n}, w\right)$ we obtain

$$
\begin{aligned}
L_{+}(\Pi) & =\left\{h_{1}^{m_{1}}\left(h_{2}^{m_{2}} \cdots\left(h_{n}^{m_{n}}(w)\right) \cdots\right) \mid m_{i} \geq 1,1 \leq i \leq n\right\} \\
& =\left\{h_{1}^{m_{1}-1}\left(h_{2}^{m_{2}-1} \cdots\left(h_{n}^{m_{n}-1}\left(h_{1}\left(\cdots\left(h_{n}(w)\right) \cdots\right)\right)\right) \cdots\right) \mid m_{i} \geq 1,1 \leq i \leq n\right\} \\
& =\left\{h_{1}^{m_{1}^{\prime}}\left(h_{2}^{m_{2}^{\prime}} \cdots\left(h_{n}^{m_{n}^{\prime}}\left(w^{\prime}\right)\right) \cdots\right) \mid m_{i}^{\prime} \geq 0,1 \leq i \leq n\right\} \\
& =L_{*}\left(\Pi^{\prime}\right)
\end{aligned}
$$

where $\Pi^{\prime}=\left(V, h_{1}, \ldots, h_{n}, w^{\prime}\right)$ with $w^{\prime}=h_{1}\left(\cdots\left(h_{n}(w)\right) \cdots\right)$.

The following example shows that the converse of Lemma 3 does not hold:

Example 4. Consider self-reproducing $\mathrm{P}$ systems over a one letter alphabet, i. e., unary P systems of the form $\Pi=\left(\{a\}, h_{1}, \ldots, h_{n}, w\right)$ as considered in more detail in the succeeding section; without loss of generality, let us assume that none of the homomorphisms $h_{i}$ with $1 \leq i \leq n$ equals the identity $h_{i}(a)=a$, because it would have no effect on the results. Clearly, the axiom $w$ belongs to the language $L_{*}(\Pi)$. Moreover, there cannot be any other string in $L_{*}(\Pi)$ that is shorter than the axiom. So, choosing $w=a^{p_{i}}$ and a sole homomorphism $h_{1}$ such that $h_{1}(a)=a^{p_{j}}$, where $p_{i}$ and $p_{j}$ are different prime numbers, we obtain a system $\Pi=\left(\{a\}, h_{1}, a^{p_{i}}\right)$ whose language $L_{*}(\Pi)$ is $\left\{a^{p_{i} p_{j}^{m}} \mid m \geq 0\right\}$. On the other hand, it is easy to see that for any other unary system $\Pi^{\prime}$ the string $a^{p_{i}}$ cannot belong to $L_{+}\left(\Pi^{\prime}\right)$ unless the axiom is $a$ and in $\Pi^{\prime}$ there must be a homomorphism $h^{\prime}$ with $h^{\prime}(a)=a^{p_{i}}$. But in this case, $a^{p_{i}^{2}}$ belongs to $L_{+}\left(\Pi^{\prime}\right)$, too. This is a contradiction, since $p_{i}$ and $p_{j}$ are different prime numbers and, thus, $a^{p_{i}^{2}}$ cannot belong to $L_{*}(\Pi)$. 


\section{Representation of unary $P$ systems}

We now consider unary $P$ systems, i. e., systems of the form

$$
\Pi=\left(\{a\}, h_{1}, \ldots, h_{n}, w\right)
$$

where $n \geq 1, w \in\{a\}^{*}$ is the axiom, and $h_{i}:\{a\} \rightarrow\{a\}^{*}, 1 \leq i \leq n$, are homomorphisms. Depending on whether or not each homomorphism has to be applied at least once, as above we distinguish two languages generated by $\Pi$ :

$$
\begin{aligned}
L_{*}(\Pi) & =\left\{h_{1}^{m_{1}}\left(h_{2}^{m_{2}} \cdots\left(h_{n}^{m_{n}}(w)\right) \cdots\right) \mid m_{i} \geq 0,1 \leq i \leq n\right\}, \\
L_{+}(\Pi) & =\left\{h_{1}^{m_{1}}\left(h_{2}^{m_{2}} \cdots\left(h_{n}^{m_{n}}(w)\right) \cdots\right) \mid m_{i} \geq 1,1 \leq i \leq n\right\} .
\end{aligned}
$$

Now we turn to a language representation which is advantageous for our purposes. To this end, we consider the decomposition of positive integers into prime factors. We denote by $F$ the set of mappings from $\mathbb{P}$ to $\mathbb{N}$ with finite support, i. e., only finitely many primes are mapped to non-zero values. Then the decompositions are represented by the mapping $p f: \mathbb{N}_{+} \rightarrow F$. For example, let $n=q_{1}^{e_{1}} \cdots q_{k}^{e_{k}}$, where $k \geq 1, q_{i}$ prime, $q_{i} \neq q_{j}$ for $i \neq j, e_{i} \geq 1,1 \leq i, j \leq k$. Then $(p f(n))\left(q_{i}\right)=e_{i}$, for $1 \leq i \leq k$, and $(p f(n))(q)=0$, for $q \notin\left\{q_{1}, \ldots, q_{k}\right\}$. For convenience, we write $p f_{n}$ for the mapping $p f(n)$. Clearly, it holds $p f_{m n}=p f_{m}+p f_{n}$. Next, let $g p f(m)$ be the index of the greatest prime factor with non-zero exponent in the decomposition of $m$, if $m>1$, and set $g p f(1)=1$.

Since $p f$ is injective, every unary language $L$ is uniquely represented by the set

$$
P(L)=\left\{p f_{m} \mid a^{m} \in L\right\} .
$$

Conversely, since $p f$ is surjective, every subset $V \subseteq F$ uniquely represents a unary language

$$
P^{-1}(V)=\left\{a^{m} \mid \text { there exists } f \in V \text { such that } m=p_{1}^{f\left(p_{1}\right)} p_{2}^{f\left(p_{2}\right)} p_{3}^{f\left(p_{3}\right)} \cdots\right\} \text {. }
$$

(Recall that $p_{i}$ always denotes the $i$-th prime number.) Alternatively, $V$ may be a set of vectors of natural numbers, e.g., $\left(m_{1}, \ldots, m_{n}\right)^{T} \in V$ implies $a^{m} \in P^{-1}(V)$, where $m=p_{1}^{m_{1}} \cdots p_{n}^{m_{n}}$. From this point of view, a vector of natural numbers may be seen as a mapping from $F$ and vice versa. In the following, we will use both notions in a synonymous way.

Let $\Pi=\left(\{a\}, w, h_{1}, \ldots, h_{n}\right)$ be a system, where $h_{i}(a)=a^{c_{i}}$, for $c_{i} \geq 1,1 \leq i \leq n$. We set $k=\max \left\{g p f(|w|), g p f\left(c_{1}\right), \ldots g p f\left(c_{n}\right)\right\}$ to be the largest prime factor with non-zero exponent appearing in $|w|$ or one of the exponents $c_{i}$, and define the matrix:

$$
M_{\Pi}=\left(\begin{array}{cccc}
p f_{c_{1}}\left(p_{1}\right) & p f_{c_{2}}\left(p_{1}\right) & \cdots & p f_{c_{n}}\left(p_{1}\right) \\
p f_{c_{1}}\left(p_{2}\right) & p f_{c_{2}}\left(p_{2}\right) & \cdots & p f_{c_{n}}\left(p_{2}\right) \\
\vdots & \vdots & \ddots & \vdots \\
p f_{c_{1}}\left(p_{k}\right) & p f_{c_{2}}\left(p_{k}\right) & \cdots & p f_{c_{n}}\left(p_{k}\right)
\end{array}\right) .
$$

Lemma 5. Let $\Pi=\left(\{a\}, h_{1}, \ldots, h_{n}, w\right)$ be a unary $P$ system. Then

$$
L_{+}(\Pi)=P^{-1}\left(M_{\Pi}\left(\mathbb{N}_{+}^{n}\right)^{T}+b\right) \quad \text { and } \quad L_{*}(\Pi)=P^{-1}\left(M_{\Pi}\left(\mathbb{N}^{n}\right)^{T}+b\right),
$$

where $b=\left(p f_{|w|}\left(p_{1}\right), p f_{|w|}\left(p_{2}\right), \ldots, p f_{|w|}\left(p_{k}\right)\right)^{T}$. 
Proof: Let $k=\max \left\{g p f(|w|), g p f\left(c_{1}\right), \ldots, g p f\left(c_{n}\right)\right\}$ and $h_{i}(a)=a^{c_{i}}$, for $c_{i} \geq 1,1 \leq i \leq n$, be defined as above. There exist $\left(m_{1}, \ldots, m_{n}\right) \in \mathbb{N}_{+}^{n}$ and $\left(e_{1}, \ldots, e_{k}\right)^{T} \in M_{\Pi}\left(\mathbb{N}_{+}^{n}\right)^{T}+b$ such that the following is true:

$$
\begin{aligned}
& a^{m} \in L_{+}(\Pi) \\
\Longleftrightarrow & a^{m}=h_{1}^{m_{1}}\left(h_{2}^{m_{2}} \cdots\left(h_{n}^{m_{n}}(w)\right) \cdots\right) \\
\Longleftrightarrow & m=|w| c_{n}^{m_{n}} c_{n-1}^{m_{n-1}} \cdots c_{1}^{m_{1}} \\
\Longleftrightarrow & p f_{m}\left(p_{i}\right)=p f_{|w|}\left(p_{i}\right)+m_{n} p f_{c_{n}}\left(p_{i}\right)+\cdots+m_{1} p f_{c_{1}}\left(p_{i}\right), \quad 1 \leq i \leq k \\
\Longleftrightarrow & p f_{m}\left(p_{i}\right)=\left(M_{\Pi}\left(m_{1}, m_{2}, \ldots, m_{n}\right)^{T}+b\right)[i], \quad 1 \leq i \leq k \\
\Longleftrightarrow & p f_{m}\left(p_{i}\right)=e_{i}, \quad 1 \leq i \leq k, \quad p f_{m}(q)=0, \quad q \notin\left\{p_{1}, \ldots, p_{k}\right\} \\
\Longleftrightarrow & m=p_{1}^{e_{1}} \cdots p_{k}^{e_{k}} \\
\Longleftrightarrow & a^{m} \in P^{-1}\left(M_{\Pi}\left(\mathbb{N}_{+}^{n}\right)^{T}+b\right) .
\end{aligned}
$$

The assertion for $L_{*}(\Pi)$ follows in an analogous way.

Due to the pumping lemma for regular languages, we immediately observe the following result:

Corollary 6. The only context-free languages that can be generated by a unary $P$ system are of the form $\left\{a^{m}\right\}$ for some $m \geq 1$.

\section{Equivalence and minimality of unary $P$ systems}

We now may take advantage of the chosen language representation in order to show the decidability of the equivalence problem for unary P systems. Moreover, we obtain a unique minimal representation.

Consider the partial ordering

$$
f \leq g \Longleftrightarrow f(p) \leq g(p) \text { for all } p \in \mathbb{P}
$$

defined on the set of mappings $\mathbb{N}^{\mathbb{P}}$. As usual, we call an element $f$ minimal if $g \leq f$ implies the identity $g=f$. Let $\Pi=\left(\{a\}, h_{1}, \ldots, h_{n}, w\right)$ be a unary $\mathrm{P}$ system and $V=M_{\Pi}\left(\mathbb{N}^{n}\right)^{T}+b$ be such that $L_{*}(\Pi)=P^{-1}(V)$. In any other system $\Pi^{\prime}=\left(\{a\}, h_{1}^{\prime}, \ldots, h_{n}^{\prime}, w^{\prime}\right)$ which generates the same language, i. e., $V^{\prime}=M_{\Pi^{\prime}}\left(\mathbb{N}^{n^{\prime}}\right)^{T}+b^{\prime}$ such that $P^{-1}\left(V^{\prime-1}\right)=P^{-1}(V)$, the same prime factors as in $\Pi$ must occur. Hence, $M_{\Pi}$ and $M_{\Pi^{\prime}}$ must have the same number of rows. Moreover, a decomposition $b=b_{1}+b_{2}$ implies $b \geq b_{1}$. Therefore, $b$ is the unique minimal element in $V$ and, thus, the unique minimal element in $V^{\prime}$, which implies $b=b^{\prime}$. For this reason, in order to deal with equivalence and minimality, for what follows we may assume $b=0$ and consequently may minimize and compare sets of the form $M_{\Pi}\left(\mathbb{N}^{n}\right)^{T}$.

An element $x \in M_{\Pi}\left(\mathbb{N}^{n}\right)^{T} \backslash\left\{0_{k}\right\}$ - where $0_{k}$ denotes the zero-vector from $\left(\mathbb{N}^{n}\right)^{T}$ - is called irreducible, if there is no decomposition of the form $x=x_{1}+x_{2}$, where $x_{1}, x_{2} \in M_{\Pi}\left(\mathbb{N}^{n}\right)^{T} \backslash\left\{0_{k}\right\}$. The next theorem leads to a unique minimal representation.

Theorem 7. Let $\Pi$ be a unary $P$ system with $n$ homomorphisms. If $M^{\prime}$ is the matrix whose columns are the $n^{\prime}$ irreducible elements of $M_{\Pi}\left(\mathbb{N}^{n}\right)^{T} \backslash\left\{0_{k}\right\}$, then

(i) all columns of $M^{\prime}$ are columns of $M_{\Pi}$ and

(ii) $M^{\prime}\left(\mathbb{N}^{n^{\prime}}\right)^{T}=M_{\Pi}\left(\mathbb{N}^{n}\right)^{T}$. 
Proof: Since an irreducible element of $M_{\Pi}\left(\mathbb{N}^{n}\right)^{T} \backslash\{0\}$ cannot be decomposed into summands, it has a representation of the form $M_{\Pi}(0, \ldots, 0,1,0, \ldots, 0)^{T}$. Therefore, it is a column of $M_{\Pi}$, and assertion (i) follows.

By (i), the inclusion $M^{\prime}\left(\mathbb{N}^{n^{\prime}}\right)^{T} \subseteq M_{\Pi}\left(\mathbb{N}^{n}\right)^{T}$ follows immediately. Now assume that in contrast to assertion (ii), the inclusion is a proper one. Then there is a minimal element $x \in M_{\Pi}\left(\mathbb{N}^{n}\right)^{T} \backslash M^{\prime}\left(\mathbb{N}^{n^{\prime}}\right)^{T}$. Since $0_{k} \in M_{\Pi}\left(\mathbb{N}^{n}\right)^{T} \cap M^{\prime}\left(\mathbb{N}^{n^{\prime}}\right)^{T}$, we have $x \neq 0_{k}$. Moreover, $x$ is not irreducible, since otherwise it would be a column of $M^{\prime}$ and, thus, would belong to $M^{\prime}\left(\mathbb{N}^{n^{\prime}}\right)^{T}$. So, there is a decomposition $x=x_{1}+x_{2}$, where $x_{1}, x_{2} \in M_{\Pi}\left(\mathbb{N}^{n}\right)^{T} \backslash\{0\}$. This implies $x_{1} \leq x$ as well as $x_{2} \leq x$. Since $x$ is minimal in $M_{\Pi}\left(\mathbb{N}^{n}\right)^{T} \backslash M^{\prime}\left(\mathbb{N}^{n^{\prime}}\right)^{T}$, both elements $x_{1}$ and $x_{2}$ belong to $M^{\prime}\left(\mathbb{N}^{n^{\prime}}\right)^{T}$. Obviously, $M^{\prime}\left(\mathbb{N}^{n^{\prime}}\right)^{T}$ is closed under addition. Therefore, $x$ belongs to $M^{\prime}\left(\mathbb{N}^{n^{\prime}}\right)^{T}$, too. From this contradiction we infer assertion (ii).

The preceding theorem shows that $M^{\prime}\left(\mathbb{N}^{n^{\prime}}\right)^{T}+b$ is the unique minimal representation of the language generated by $\Pi$, i.e., $L_{*}(\Pi)=P^{-1}\left(M^{\prime}\left(\mathbb{N}^{n^{\prime}}\right)^{T}+b\right)$. It is easy to determine the irreducible columns out of the $n$ columns of $M_{\Pi}$. Hence, matrix $M^{\prime}$ can effectively be constructed from $M_{\Pi}$. Conversely, given some $M^{\prime}$ with $n^{\prime}$ columns and $b$, the unary $\mathrm{P}$ system $\Pi^{\prime}$ whose language is $P^{-1}\left(M^{\prime}\left(\mathbb{N}^{n^{\prime}}\right)^{T}+b\right)$ can effectively be constructed, too.

Corollary 8. There exists an effective algorithm which minimizes the number of homomorphisms (membranes) of a unary $P$ system.

Corollary 9. The equivalence of unary $P$ systems is decidable.

\section{Descriptional complexity of unary P systems}

This section is devoted to descriptional complexity issues of unary $\mathrm{P}$ systems with a different number of homomorphisms (membranes). The key question is how succinct a language, given by some unary $\mathrm{P}$ system with $n$ homomorphisms, can be represented by some unary $\mathrm{P}$ system with at least $n$ homomorphisms.

In order to talk about the economy of descriptions we first have to define what is meant by the size of a system. In general, we are interested to measure the length of the string that defines a system. In particular, we use more convenient size measures, such that there is a recursive upper bound for the length of the defining string depending on the chosen size measure. For example, the size of a finite automaton equals the product of the number of its states and the number of its input symbols.

The size $|\Pi|$ of a unary $\mathrm{P}$ system $\Pi=\left(\{a\}, h_{1}, \ldots, h_{n}, w\right)$ is defined to be

$$
|\Pi|=|w|+\sum_{1 \leq i \leq n}\left|h_{i}(a)\right|
$$

We denote the family of unary $\mathrm{P}$ systems by $\mathcal{F}$. Clearly, the considered measure implies a total, recursive function mapping a unary $\mathrm{P}$ system to its size, such that $\mathcal{F}$ is recursively enumerable in order of increasing size, and does not contain infinitely many members of the same size.

Let $\mathcal{F}_{1}$ and $\mathcal{F}_{2}$ be two subfamilies of $\mathcal{F}$. A function $f: \mathbb{N}_{+} \rightarrow \mathbb{N}_{+}$, with $f(n) \geq n$, is said to be an upper bound for the increase in size when changing from a minimal description in $\mathcal{F}_{1}$ to an equivalent minimal description in $\mathcal{F}_{2}$, if

$$
\min \left\{|\Pi| \mid \Pi \in \mathcal{F}_{2} \text { generates } L\right\} \leq f\left(\min \left\{|\Pi| \mid \Pi \in \mathcal{F}_{1} \text { generates } L\right\}\right)
$$

for all languages $L$ generated by some system in $\mathcal{F}_{1}$ as well as by some system in $\mathcal{F}_{2}$. 
The following theorem is an immediate consequence of Theorem 7 Since any matrix of some representation contains at least the columns of irreducible elements, any equivalent system has at least the homomorphisms associated with these columns, respectively.

Theorem 10. Let $\Pi$ be a unary $P$ system with $n$ homomorphisms where

$$
L_{*}(\Pi)=P^{-1}\left(M_{\Pi}\left(\mathbb{N}^{n}\right)^{T}+b\right) .
$$

If the columns of $M_{\Pi}$ are different irreducible elements of $M_{\Pi}\left(\mathbb{N}^{n}\right)^{T} \backslash\left\{0_{k}\right\}$, then every unary system $\Pi^{\prime}$ with $L_{*}(\Pi)=L_{*}\left(\Pi^{\prime}\right)$ is at most of size $|\Pi|$.

So, in case of $L_{*}(\Pi)$, the identity is an upper bound for the increase of size when possibly changing from a description with $n$ homomorphisms to an equivalent description with $n^{\prime}<n$ homomorphisms. The situation is different in case of $L_{+}(\Pi)$ :

Theorem 11. Let $\Pi$ be a unary $P$ system with $n$ homomorphisms. If there is a unary $P$ system $\Pi^{\prime}$ with $n-1$ homomorphisms such that $L_{+}(\Pi)=L_{+}\left(\Pi^{\prime}\right)$, then $\left|\Pi^{\prime}\right| \in O\left(|\Pi|^{2}\right)$.

Proof: If a language $L=L_{+}(\Pi)$ is generated by some unary $\mathrm{P}$ system

$$
\Pi=\left(\{a\}, h_{1}, \ldots, h_{n}, w\right)
$$

whose number of homomorphisms can be reduced, then there is at least one column of $M_{\Pi}$ which is not an irreducible element of $M_{\Pi}\left(\mathbb{N}^{n}\right)^{T} \backslash\left\{0_{k}\right\}$. Let $h_{n}$ be the associated homomorphism, then

$$
\Pi^{\prime}=\left(\{a\}, h_{1}, \ldots, h_{n-1}, h_{n}(w)\right)
$$

generates $L$, too, i. e., $L=L_{+}\left(\Pi^{\prime}\right)$. Therefore, we can approximate the size of $\Pi^{\prime}$ as follows:

Let $m=\max \left\{\left|h_{i}(a)\right| \mid 1 \leq i \leq n\right\}$, then

$$
\begin{aligned}
\left|\Pi^{\prime}\right| & =|\Pi|-\left|h_{n}(a)\right|-|w|+\left|h_{n}(w)\right| \\
& \leq|\Pi|-\left|h_{n}(a)\right|-|w|+m|w| \\
& \leq|\Pi|-1+(m-1)|w| \\
& \leq|\Pi|-1+(|\Pi|-1) *|\Pi| \\
& =|\Pi|^{2}-1 \in O\left(|\Pi|^{2}\right) .
\end{aligned}
$$

The next lemma shows that the upper bound of Theorem 11 can be reached.

Lemma 12. Let $\Pi=\left(\{a\}, h_{1}, h_{2}, w\right)$ where $h_{1}(a)=h_{2}(a)=a^{m}$ and $w=a^{m}$, for some $m \geq 2$, then any unary $P$ system $\Pi^{\prime}$ with $L_{+}(\Pi)=L_{+}\left(\Pi^{\prime}\right)$ has size $\Omega\left(|\Pi|^{2}\right)$.

Proof: Obviously, $h_{1}(a)=a^{m}$ is associated with a necessary column in $M_{\Pi}$. So, the sole equivalent unary $\mathrm{P}$ system with one homomorphism is

$$
\Pi^{\prime}=\left(\{a\}, h_{1}, h_{2}(w)\right) .
$$

For the sizes we obtain $|\Pi|=3 m$ and $\left|\Pi^{\prime}\right|=m+m^{2} \in \Omega\left(|\Pi|^{2}\right)$. 
If we reduce the number of homomorphisms by more than one, i. e., we iterate the construction $x$ times, then we do not obtain sizes of the form $O\left(|\Pi|^{2^{x}}\right)$. The reason is that we have to multiply the sizes by at most a factor $|\Pi|$ in each step.

Corollary 13. Let $\Pi$ be a unary $P$ system with $n$ homomorphisms. If there is a unary $P$ system $\Pi^{\prime}$ with $n-x$ homomorphisms such that $L_{+}(\Pi)=L_{+}\left(\Pi^{\prime}\right)$, then $\left|\Pi^{\prime}\right| \in O\left(|\Pi|^{x+1}\right)$.

By an immediate generalization of the proof of Lemma 12 we obtain a matching lower bound (in the order of magnitude) in the worst case.

Lemma 14. Let $\Pi=\left(\{a\}, h_{1}, \ldots, h_{n}, w\right)$ where $h_{1}(a)=\cdots=h_{n}(a)=a^{m}$ and $w=a^{m}$, for some $m \geq 2$, then any unary $P$ system $\Pi^{\prime}$ with $n-x$ homomorphisms, $1 \leq x \leq n-1$, and $L_{+}(\Pi)=L_{+}\left(\Pi^{\prime}\right)$ has size $\Omega\left(|\Pi|^{x+1}\right)$.

Finally, if we fix the number $m$ and consider arbitrarily large $n$, then the trade-off in the size may become exponential with respect to the base $m$.

Example 15. Let $\Pi=\left(\{a\}, h_{1}, \ldots, h_{n}, w\right)$ where $h_{1}(a)=\cdots=h_{n}(a)=a^{m}$ and $w=a^{m}$, for some $m \geq 2$, then any unary P system $\Pi^{\prime}$ with one homomorphism and $L_{+}(\Pi)=L_{+}\left(\Pi^{\prime}\right)$ has size $\Omega\left(|\Pi|^{n}\right)$.

The representation and the descriptional complexity of languages generated by less restricted variants of $\mathrm{P}$ systems remains as a challenging task for future research; for example, we may ask to which extent the results proved above for unary $\mathrm{P}$ systems can also be formulated for self-reproducing $\mathrm{P}$ systems.

\section{References}

[1] J. Dassow (1986): On Compound Lindenmayer Systems. In: A. Salomaa G. Rozenberg, editor: The Book of L. Springer-Verlag, Berlin, pp. 75-86.

[2] J. Dassow, G. Păun, G. Thierrin \& S. Yu (2001): Tree-Systems of Morphisms. Acta Informatica 38, pp. $131-153$

[3] R. Freund (2001): Special Variants of P Systems Inducing an Infinite Hierarchy with Respect to the Number of Membranes. Bulletin of the EATCS 75, pp. 209-219.

[4] G. Păun (2000): Computing with membranes. J. of Computer and System Sciences 61, pp. 108-143. Also TUCS Research Report, 208, 1998 http://www.tucs.fi.

[5] G. Păun (2002): Membrane Computing. An Introduction. Springer-Verlag, Berlin.

[6] G. Păun \& G. Rozenberg (2002): A Guide to Membrane Computing. Theoretical Computer Science 287, pp. 73-100.

[7] G. Păun, Y. Sakakibara \& T. Yokomori (2002): P Systems on Graphs of Restricted Forms. Publicationes Mathematicae Debrecen 60, pp. 635-660.

[8] G. Rozenberg \& A. Salomaa, editors (1997): Handbook of Formal Languages (3 volumes). Springer-Verlag, Berlin.

[9] The P Systems Web Page http://ppage.psystems .eu. 